\title{
Correlation between mean transverse momentum and anisotropic flow in heavy-ion collisions
}

\author{
Giuliano Giacalone, ${ }^{1}$ Fernando G. Gardim, ${ }^{2}$ Jacquelyn Noronha-Hostler, ${ }^{3}$ and Jean-Yves Ollitrault ${ }^{1}$ \\ ${ }^{1}$ Université Paris Saclay, CNRS, CEA, Institut de physique théorique, 91191 Gif-sur-Yvette, France \\ ${ }^{2}$ Instituto de Ciência e Tecnologia, Universidade Federal de Alfenas, 37r15-400 Poços de Caldas, MG, Brazil \\ ${ }^{3}$ Department of Physics, University of Illinois at Urbana-Champaign, Urbana, IL 61801, USA
}

(Dated: February 23, 2021)

\begin{abstract}
The correlation between the mean transverse momentum of outgoing particles, $\left\langle p_{t}\right\rangle$, and the magnitude of anisotropic flow, $v_{n}$, has recently been measured in $\mathrm{Pb}+\mathrm{Pb}$ collisions at the CERN Large Hadron Collider, as a function of the collision centrality. We confirm the previous observation that event-by-event hydrodynamics predicts a correlation between $v_{n}$ and $\left\langle p_{t}\right\rangle$ that is similar to that measured in data. We show that the magnitude of this correlation can be directly predicted from the initial condition of the hydrodynamic calculation, for $n=2,3$, if one replaces $v_{n}$ by the corresponding initial-state anisotropy, $\varepsilon_{n}$, and $\left\langle p_{t}\right\rangle$ by the total energy per unit rapidity of the fluid at the beginning of the hydrodynamic expansion.
\end{abstract}

\section{INTRODUCTION}

Anisotropic flow has been much studied in ultrarelativistic heavy-ion collisions, as it probes the properties of the little quark-gluon plasma formed in these collisions [1]. The event-by-event fluctuations of $v_{n}$, the $n^{\text {th }}$ Fourier harmonic of the azimuthal distribution of the emitted hadrons, have been precisely characterized [24, as well as the mutual correlations between different flow harmonics 5, 8]. Recently, following a suggestion by Bożek 9], the ATLAS Collaboration has measured a correlation of a new type, namely, the correlation between the mean transverse momentum, $\left\langle p_{t}\right\rangle$, and $v_{n}^{2}[10$, a quantity dubbed $\rho_{n}$. It is the first time that anisotropic flow, which is dimensionless, is correlated with a dimensionful quantity. This opens a new window on the study of $v_{n}$, by giving new information about its correlation with the size and/or temperature of the quark-gluon plasma. Although event-by-event hydrodynamic results on $\rho_{n}$ are in fair agreement with experimental data [1], a clear picture of the physical mechanism that produces this correlation is still missing. In this paper, we explain the origin of the correlation between $\left\langle p_{t}\right\rangle$ and $v_{n}$ in hydrodynamic calculations. We first confirm, in Sec. II that state-of-the-art hydrodynamic calculations yield results on $\rho_{n}$ that are in agreement with recent $\mathrm{Pb}+\mathrm{Pb}$ data. We use results from hydrodynamic calculations [12] obtained prior to the ATLAS analysis, so that they can be considered as predictions. We then unravel, in Sec. III. the physical mechanism behind $\rho_{n}$. While it is well established that $v_{2}$ and $v_{3}$ originate on an event-by-event basis from the anisotropies of the initial density profile, $\varepsilon_{n}[13$ [18, the new crucial feature that we shall elucidate here is the origin of $\left\langle p_{t}\right\rangle$ fluctuations in hydrodynamics. These fluctuations are thought to be driven by fluctuations in the fireball size, $R[19,20$, a phenomenon referred to as size-flow transmutation [21. We show in Sec. III A that, in fact, a much better predictor for $\left\langle p_{t}\right\rangle$ is provided by the initial total energy per rapidity of the fluid [22]. On this basis, in Sec. IIIB we evaluate $\rho_{n}$ using a standard initial-state model for $\mathrm{Pb}+\mathrm{Pb}$ collisions, and we obtain results that are in good agreement with ATLAS data. We further show that agreement with data is instead lost if one uses $R$ as an event-by-event predictor of $\left\langle p_{t}\right\rangle$.

\section{RESULTS FROM EVENT-BY-EVENT HYDRODYNAMICS}

The ATLAS Collaboration measured the Pearson correlation coefficient between the mean transverse momentum and the anisotropic flow of the event [10]. Experimentally, this is obtained from a three-particle correlation introduced by Bożek 9. Since self correlations are subtracted in the measure of the correlation coefficient (i.e., one does not correlate a particle with itself), this observable is insensitive to trivial statistical fluctuations and probes genuine dynamical fluctuations [23], due to correlations. In hydrodynamics, $\rho_{n}$ can be evaluated as:

$$
\rho_{n} \equiv \frac{\left\langle\left\langle p_{t}\right\rangle v_{n}^{2}\right\rangle-\left\langle\left\langle p_{t}\right\rangle\right\rangle\left\langle v_{n}^{2}\right\rangle}{\sigma_{p_{t}} \sigma_{v_{n}^{2}}},
$$

where, following the notation of Ref. 20], $\left\langle p_{t}\right\rangle$ denotes an average over the single-particle momentum distribution, $f(p)$, at freeze-out in a given event ${ }^{1}$, and the outer angular brackets denote an average over events in a given multiplicity (centrality) window. $\sigma_{p_{t}}$ and $\sigma_{v_{n}}$ denote, respectively, the standard deviation of $\left\langle p_{t}\right\rangle$ and of $v_{n}^{2}$ :

$$
\begin{aligned}
\sigma_{p_{t}} & \equiv \sqrt{\left\langle\left\langle p_{t}\right\rangle^{2}\right\rangle-\left\langle\left\langle p_{t}\right\rangle\right\rangle^{2}}, \\
\sigma_{v_{n}^{2}} & \equiv \sqrt{\left\langle v_{n}^{4}\right\rangle-\left\langle v_{n}^{2}\right\rangle^{2}} .
\end{aligned}
$$

We evaluate $\rho_{n}$ using hydrodynamic simulations. The setup of our calculation is the same as in Ref. [12.

\footnotetext{
${ }^{1}$ Using this notation, one can also write $v_{n} \equiv\left|\left\langle e^{i n \varphi}\right\rangle\right|$, where $\varphi$ is the azimuthal angle of the particle momentum.
} 


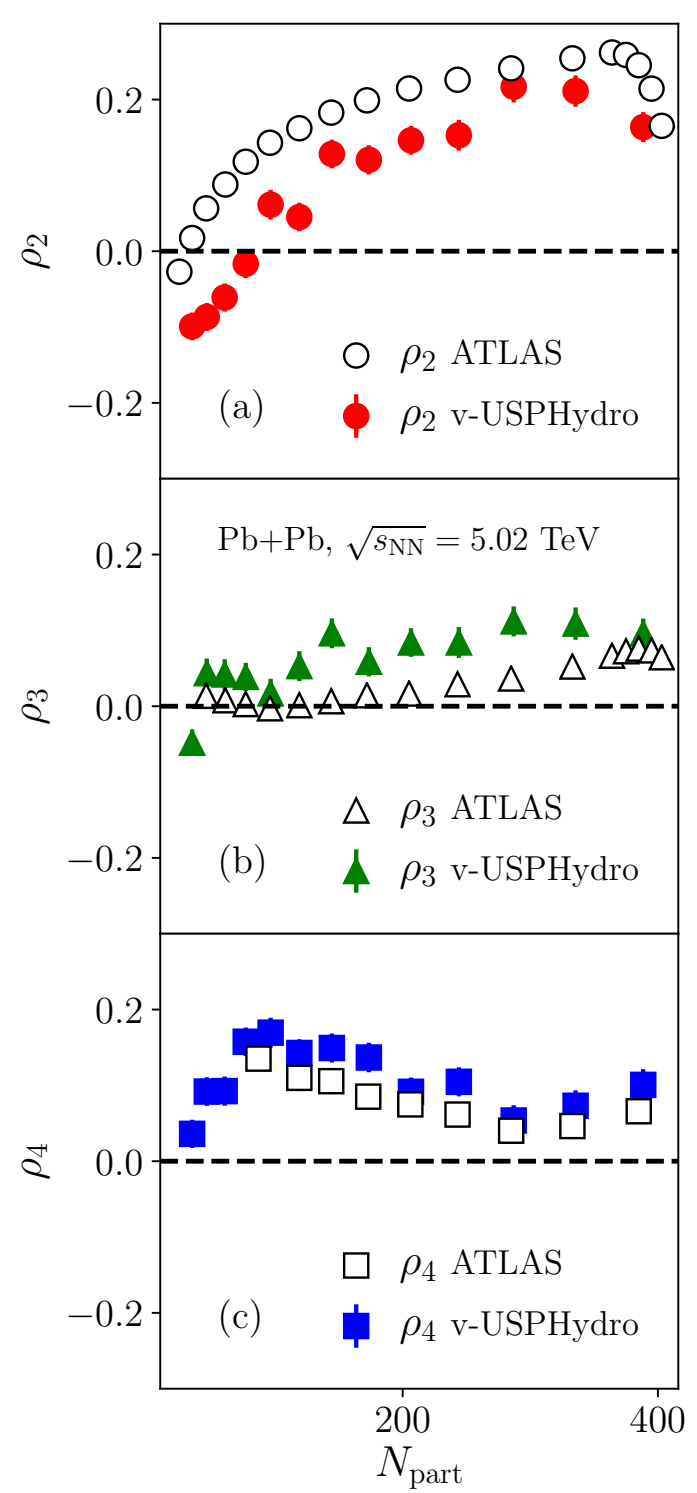

FIG. 1. (Color online) Value of $\rho_{n}$ for $n=2$ (a), $n=3$ (b), $n=4$ (c), as a function of the number of participant nucleons in $\mathrm{Pb}+\mathrm{Pb}$ collisions at $\sqrt{s_{\mathrm{NN}}}=5.02 \mathrm{TeV}$. Empty symbols are experimental results from the ATLAS Collaboration [10, integrated over $0.5<p_{t}<2 \mathrm{GeV} / \mathrm{c}$, while full symbols are hydrodynamic results 12 , integrated over $0.2<p_{t}<3 \mathrm{GeV} / \mathrm{c}$.

We evolve 50000 minimum bias $\mathrm{Pb}+\mathrm{Pb}$ collisions at $\sqrt{s_{\mathrm{NN}}}=5.02 \mathrm{TeV}$ through the $2+1$ viscous relativistic hydrodynamical code V-USPHYDRO 24,26. The initial condition of the evolution is the profile of entropy density generated using the $\mathrm{T}_{\mathrm{R}} \mathrm{ENT}$ To model [27, tuned as in Ref. 28. 2 We neglect the expansion of the system during the pre-equilibrium phase [29] 31, and start

\footnotetext{
2 That is, we implement a geometric average of nuclear thickness functions (parameter $p=0$ ), where the thickness function of a nucleus is given by a linear superimposition of the thicknesses
}

hydrodynamics at time $\tau_{0}=0.6 \mathrm{fm} / \mathrm{c}$ after the collision 32. We use an equation of state based on lattice QCD [33, and we implement a constant shear viscosity over entropy ratio $\eta / s=0.047$ [34]. Fluid cells are transformed into hadrons [35] when the local temperature drops below $150 \mathrm{MeV}$. All hadronic resonances can be formed during this freeze-out process, and we implement subsequent strong decays into stable hadrons. To mimic the centrality selection performed in experiment, we sort events into centrality classes according to their initial entropy ( $5 \%$ classes are used). We evaluate hadron observables by integrating over the transverse momentum range $0.2<p_{t}<3 \mathrm{GeV} / \mathrm{c}$, and over $|\eta|<0.8$.

In Fig. 1 we show our results along with ATLAS data. The kinematic cuts in $p_{t}$ and $\eta$ in our hydrodynamic calculation differ from those of ATLAS. The reason is that we use results from a prior high-statistics hydrodynamic calculations [12. ATLAS shows three sets of results, with two lower cuts $(0.5$ and $1 \mathrm{GeV} / \mathrm{c})$ and two upper cuts $(2$ and $5 \mathrm{GeV} / \mathrm{c})$ in $p_{t}$. Since hydrodynamics is meant to explain the production of particles at low- $p_{t}$, we choose the lowest values for both cuts, that is, data integrated over $0.5<p_{t}<2 \mathrm{GeV} / \mathrm{c}$. The calculation is in reasonable agreement with data, and we conclude that event-by-event relativistic hydrodynamics captures semi-quantitatively the magnitude and the centrality dependence of $\rho_{2}, \rho_{3}$ and $\rho_{4}$.

\section{PHYSICAL ORIGIN OF $\rho_{2}$ AND $\rho_{3}$}

\section{A. Initial energy as a predictor for $\left\langle p_{t}\right\rangle$}

The full hydrodynamic calculation allows us to reproduce the experimental data, but it does not give much insight into the physics underlying the observed $\rho_{n}$. In this Section, we trace the origin of $\rho_{n}$ back to the initial state of the hydrodynamic calculation. It is well known that $v_{2}$ and $v_{3}$ are driven by the initial spatial eccentricity, $\varepsilon_{2}$, and triangularity, $\varepsilon_{3}$, on an event-by-event basis. The other ingredient entering $\rho_{n}$ is the mean transverse momentum of the event. Therefore, we must identify the property of the initial state which drives the mean transverse momentum on an event-by-event basis.

It has recently been shown 22 that if one fixes the total entropy (which in an experiment amounts to fixing the centrality of the collision), then $\left\langle p_{t}\right\rangle$ is essentially determined by the energy of the fluid per unit rapidity at the initial time $\tau_{0}$, which we denote by $E_{i}$ :

$$
E_{i} \equiv \tau_{0} \int \epsilon\left(\tau_{0}, x, y\right) d x d y
$$

of the corresponding participant nucleons, modeled as Gaussian density profiles of width $w=0.51 \mathrm{fm}$. The thickness of each participant nucleon is further allowed to fluctuate in normalization according to a gamma distribution of unit mean and standard deviation $1 / \sqrt{k}$, with $k=1.6$. 


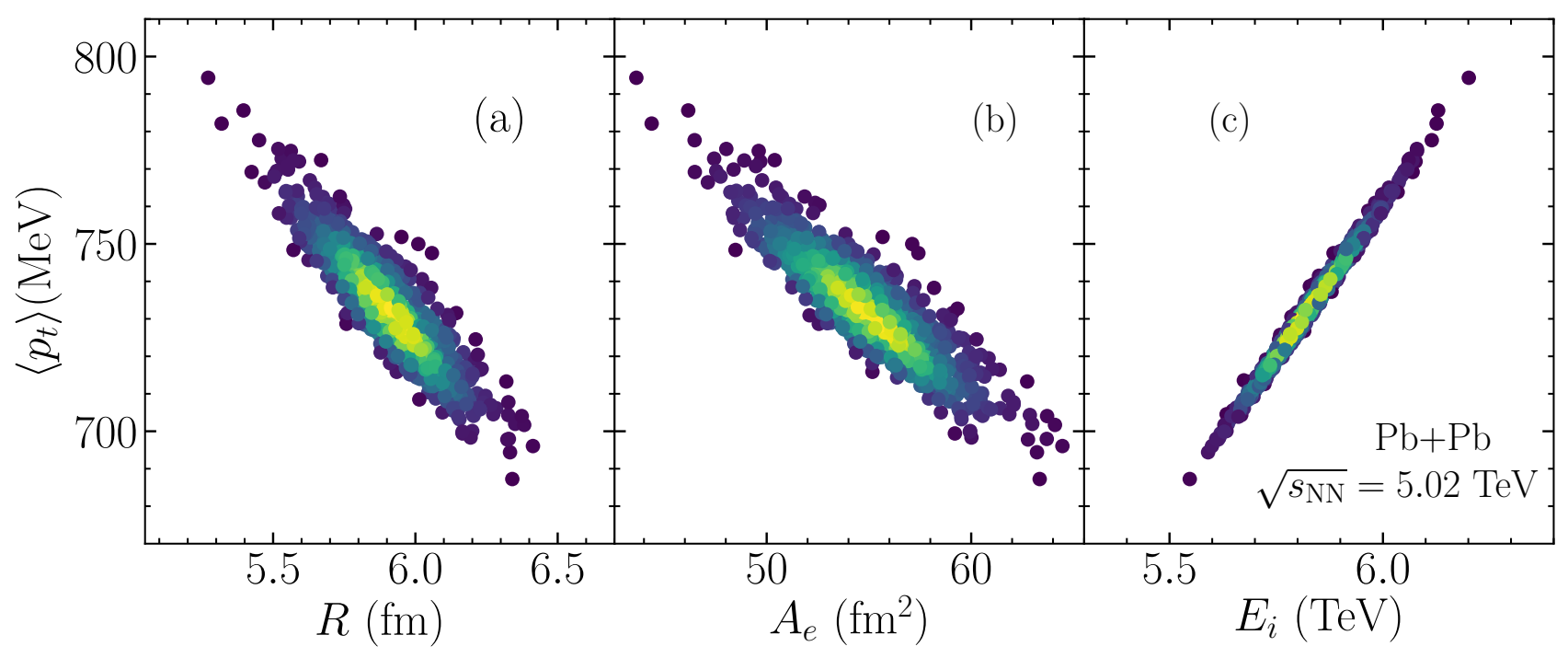

FIG. 2. (Color online) Results from ideal hydrodynamic simulations of $\mathrm{Pb}+\mathrm{Pb}$ collisions at $\sqrt{s_{\mathrm{NN}}}=5.02 \mathrm{TeV}$ at impact parameter $b=2.5 \mathrm{fm}$. 850 events have been simulated, where each event has the same total entropy, but a different entropy density profile. Each symbol represents a different event. (a) Scatter plot of the mean transverse momentum of charged particles, $\left\langle p_{t}\right\rangle$, versus the initial size, $R$, defined by Eq. (4). (b) Scatter plot $\left\langle p_{t}\right\rangle$, versus the elliptic area $A_{e}$, defined by Eq. (5). (c) Scatter plot of $\left\langle p_{t}\right\rangle$ versus the initial energy per unit rapidity, $E_{i}$, defined by Eq. (3).

where $\epsilon$ is the energy density, and the integral runs over the transverse plane. This is at variance with the earlier claims [19] that $\left\langle p_{t}\right\rangle$ is determined by the initial transverse size of the fireball, $R$, defined as [20, 21]:

$$
R^{2} \equiv 2 \frac{\int\left(x^{2}+y^{2}\right) s\left(\tau_{0}, x, y\right) d x d y}{\int s\left(\tau_{0}, x, y\right) d x d y}
$$

where $s$ is the entropy density ${ }^{3}$ Schenke, Shen and Teaney [36] have recently proposed an improved predictor of $\left\langle p_{t}\right\rangle$, relative to (4). Their idea is that the relevant quantity is the area of the overlap region, and that the elliptical shape must be taken into account in evaluating this area, which is defined as Eq. (34) of [36]:

$$
A_{e} \equiv \frac{\pi}{2} R^{2} \sqrt{1-\varepsilon_{2}^{2}}
$$

where $\varepsilon_{2}$ is the initial eccentricity.

To illustrate the difference between these three predictors, we have evaluated $E_{i}, R, A_{e}$ and $\left\langle p_{t}\right\rangle$ in eventby-event hydrodynamics at fixed initial entropy. Note that the minimum bias calculation performed in Sec. II is not well suited for this purpose, as, even if we narrowed down the width of our centrality bins by a factor 2 , there would still be significant entropy fluctuations in our sample. For this reason, we resort to the calculation shown in Ref. [22]. Here the events are evaluated at fixed impact

\footnotetext{
3 The factor 2 ensures that for a uniform entropy density $s\left(\tau_{0}, x, y\right)$ in a circle of radius $R$, the right-hand side gives $R^{2}$.
}

parameter $b=2.5 \mathrm{fm}$, and at fixed total entropy corresponding to the mean entropy of $\mathrm{Pb}+\mathrm{Pb}$ collisions at $\sqrt{s_{N N}}=5.02 \mathrm{TeV}$ in the $0-5 \%$ centrality window. Also, we perform an ideal hydrodynamic expansion, which ensures conservation of entropy. The initial condition of the calculation, the equation of state, and the initialization time, $\tau_{0}$, are the same used in the calculation of Sec. $\mathrm{II}^{4}$ This calculation is evolved through the MUSIC hydrodynamic code 38 40].

Figure 2(a) displays the scatter plot of $R$ vs. $\left\langle p_{t}\right\rangle$ obtained in this calculation. These two quantities are negatively correlated, as already shown by other authors 21 . The explanation is that for a fixed total entropy, a smaller size generally implies a larger entropy density, hence a larger temperature, which in turn implies a larger $\left\langle p_{t}\right\rangle$ [1]. There is, however, a significant spread of the values of $\left\langle p_{t}\right\rangle$ for a fixed $R$. A similar spread is observed if one correlates $\left\langle p_{t}\right\rangle$ with the elliptic area, $A_{e}$, as shown in Fig. 2(b) ${ }^{5}$ By contrast, there is an almost one-to-one correspondence between $\left\langle p_{t}\right\rangle$ and the initial energy, $E_{i}$, as displayed in Fig. 2 (b). We shall show in Sec. IIIB that, in order to understand the measured correlation

\footnotetext{
4 The sole differences are that we implement slightly smaller entropy fluctuations, using $k=2$ in $\mathrm{T}_{\mathrm{R}}$ ENTo, which provide a better fit of LHC data [12, as well as a slightly higher freeze-out temperature $T_{f}=156.5 \mathrm{MeV}$, which has now become a more standard choice 37 .

5 Note that we consider fairly central collisions where $\varepsilon_{2}$ is small. This explains the similar correlation patterns in Figs. 2 2a) and 2 (b). The situation would likely be different in mid-central collisions where $\varepsilon_{2}$ is larger.
} 
between $\left\langle p_{t}\right\rangle$ and $v_{n}$, it is indeed crucial to employ $E_{i}$ as a predictor of the average transverse momentum.

\section{B. Results from models of initial conditions}

We first explain how $\rho_{n}$, defined in Eq. (1), can be evaluated from the initial conditions of the hydrodynamic calculations. First, one uses the approximate proportionality between $v_{n}$ and the initial anisotropy $\varepsilon_{n}$ [17] for $n=2,3$ :

$$
v_{n}=\kappa_{n} \varepsilon_{n},
$$

where $\kappa_{n}$ is a response coefficient which is the same for all events at a given centrality 6

Next, one uses the observation, made in Sec. III A, that $\left\langle p_{t}\right\rangle$ is a function of the initial energy $E_{i}$ for a fixed total entropy. We first relax the stringent condition that the total entropy $S$ is fixed. Since $\left\langle p_{t}\right\rangle$ is the average transverse momentum per particle, the relevant predictor is also the initial energy per particle, which is proportional to $E_{i} / S$ since the entropy is proportional to the number of particles. We therefore replace $E_{i}$ with $E_{i} / S$ from now on 7 and we assume that $\left\langle p_{t}\right\rangle=f\left(E_{i} / S\right)$, where $f\left(E_{i} / S\right)$ is some smooth function of $E_{i} / S$.

Linearizing in the fluctuations of $E_{i} / S$ and $\left\langle p_{t}\right\rangle$ around their mean values, $\left\langle E_{i} / S\right\rangle$ and $\left\langle\left\langle p_{t}\right\rangle\right\rangle$, one obtains

$$
\left\langle p_{t}\right\rangle-\left\langle\left\langle p_{t}\right\rangle\right\rangle=f^{\prime}\left(\left\langle E_{i} / S\right\rangle\right)\left(\frac{E_{i}}{S}-\left\langle\frac{E_{i}}{S}\right\rangle\right) .
$$

Inserting Eqs. (6) and (7) into Eq. (1), one obtains:

$$
\rho_{n}=\frac{\left\langle\frac{E_{i}}{S} \varepsilon_{n}^{2}\right\rangle-\left\langle\frac{E_{i}}{S}\right\rangle\left\langle\varepsilon_{n}^{2}\right\rangle}{\sigma_{E_{i} / S} \sigma_{\varepsilon_{n}^{2}}} \frac{f^{\prime}\left(\left\langle E_{i} / S\right\rangle\right)}{\left|f^{\prime}\left(\left\langle E_{i} / S\right\rangle\right)\right|},
$$

where $\sigma_{E_{i} / S}$ and $\sigma_{\varepsilon_{n}^{2}}$ denote the standard deviations, obtained by replacing $\left\langle p_{t}\right\rangle$ and $v_{n}$ with $E_{i} / S$ and $\varepsilon_{n}$ in Eq. 21. Remarkably enough, the dependence on the unknown function $f\left(E_{i} / S\right)$ cancels, except for the sign of $f^{\prime}\left(\left\langle E_{i} / S\right\rangle\right)$. An important advantage of Eq. (8) is that it allows us to evaluate $\rho_{n}$ in millions of simulated initial conditions with little computational effort. We have generated 20 million minimum bias $\mathrm{Pb}+\mathrm{Pb}$ events using the same $\mathrm{T}_{\mathrm{R}}$ ENTo parametrization as in Fig. 2. We sort the events into narrow $0.25 \%$ centrality bins, and in each bin we evaluate $\rho_{n}$ according to Eq. (8). To evaluate $E_{i}$ in each event, we assume that the entropy profile returned by $\mathrm{T}_{\mathrm{R}} \mathrm{ENTo}, s$, is related to the energy density,

\footnotetext{
6 This linear response works for $v_{2}$ and $v_{3}$, but not for $v_{4}$ [16], so that we do not discuss $\rho_{4}$ in this section.

7 In practice, the results presented below use very narrow entropy (centrality) bins, so that one would obtain the same results using $E_{i}$ or $E_{i} / S$. If one uses $E_{i} / S$, results are unchanged if one uses wider centrality bins, up to $2 \%$. A moderate variation starts to be visible if one uses $5 \%$ bins, as with other observables 42 .
}

$\epsilon$, of the event through $\epsilon \propto s^{4 / 3}$. This is typically a very good approximation at the high temperatures achieved in the initial state of nucleus-nucleus collisions. Our result is displayed in Fig. 3 as a shaded band. Note that we recombine $0.25 \%$ bins into $1 \%$ bins for sake of visualization. Our TRENTo calculation is in good agreement with ATLAS data (open symbols) for both $\rho_{2}$ and $\rho_{3}$, and is consistent with the full hydrodynamic calculation shown in Fig. 1, in the sense that both evaluations slightly underestimates $\rho_{2}$ while they overestimates $\rho_{3}$.

Note that $\rho_{2}$ and $\rho_{3}$ measured by the ATLAS Collaboration have a slight dependence on the $p_{t}$ cut used in the analysis [10]. The difference between our results and experimental data is of the same order, or smaller, than the dependence of experimental results on the $p_{t}$ cuts. This feature is not captured by our prediction, which is independent of these cuts by construction. It would be therefore interesting to have new measurements of $\rho_{n}$ with a lower $p_{t}$ cut, of order 0.2 or $0.3 \mathrm{GeV} / \mathrm{c}$, which is where the bulk of the produced particles sits. This may improve agreement between our evaluations and data. It would also be interesting to have a full, high-statistics calculation of $\rho_{n}$ in hydrodynamics to study the deviations from the predictor Eq. (8), and the dependence on the $p_{t}$ cut. Note that our initial-state predictor (8) might have a broader range of applicability than hydrodynamics itself It is already well known that the hypothesis that $v_{n}$ is proportional to $\varepsilon_{n}$ is more general than hydrodynamics [43, 44]. In the same way, our hypothesis that $\left\langle p_{t}\right\rangle$ is determined by the initial energy seems a natural consequence of conservation laws, and might still be valid when hydrodynamics is not.

While the quantitative results shown in Fig. 3 depend on the parametrization of the $\mathrm{T}_{\mathrm{R}} \mathrm{ENT}$ To model, we show in Appendix A that the main qualitative features, for instance the fact that $\rho_{n}$ is positive in central collisions, are robust and model-independent. It is interesting though that the choice of parameters made here, namely, $p=0$, preferred from previous comparisons [18, 28, and $k=2$ [12, also optimizes agreement with $\rho_{n}$ data.

Finally, we compare with results obtained with two different predictors of $\left\langle p_{t}\right\rangle$ introduced in Sec. III A The initial size $R$ [20], and the entropy per unit area $S / A_{e}$ 36]. We thus evaluate $\rho_{n}$ by replacing $E_{i} / S$ with $1 / R^{9}$ or $S / A_{e}$ in Eq. 8. With $1 / R$, both $\rho_{2}$ and $\rho_{3}$ are completely different (dashed lines in Fig. 3). These results show that the correlation between $\left\langle p_{t}\right\rangle$ and $v_{n}^{2}$ is not driven by the event-by-event fluctuations of the fireball size. The predictor $S / A_{e}$ (dotted lines in Fig. 3 ) gives results similar to $E_{i} / S$ for $\rho_{2}$, not for $\rho_{3}$. None of these alternative predictors allows one to reproduce the observed $\rho_{2}$ and $\rho_{3}$.

\footnotetext{
8 We thank the anonymous referee for suggesting this.

${ }^{9}$ We use $1 / R$ instead of $R$ because the correlation between $R$ and $\left\langle p_{t}\right\rangle$ is negative, as shown in Fig. 2 (a).
} 

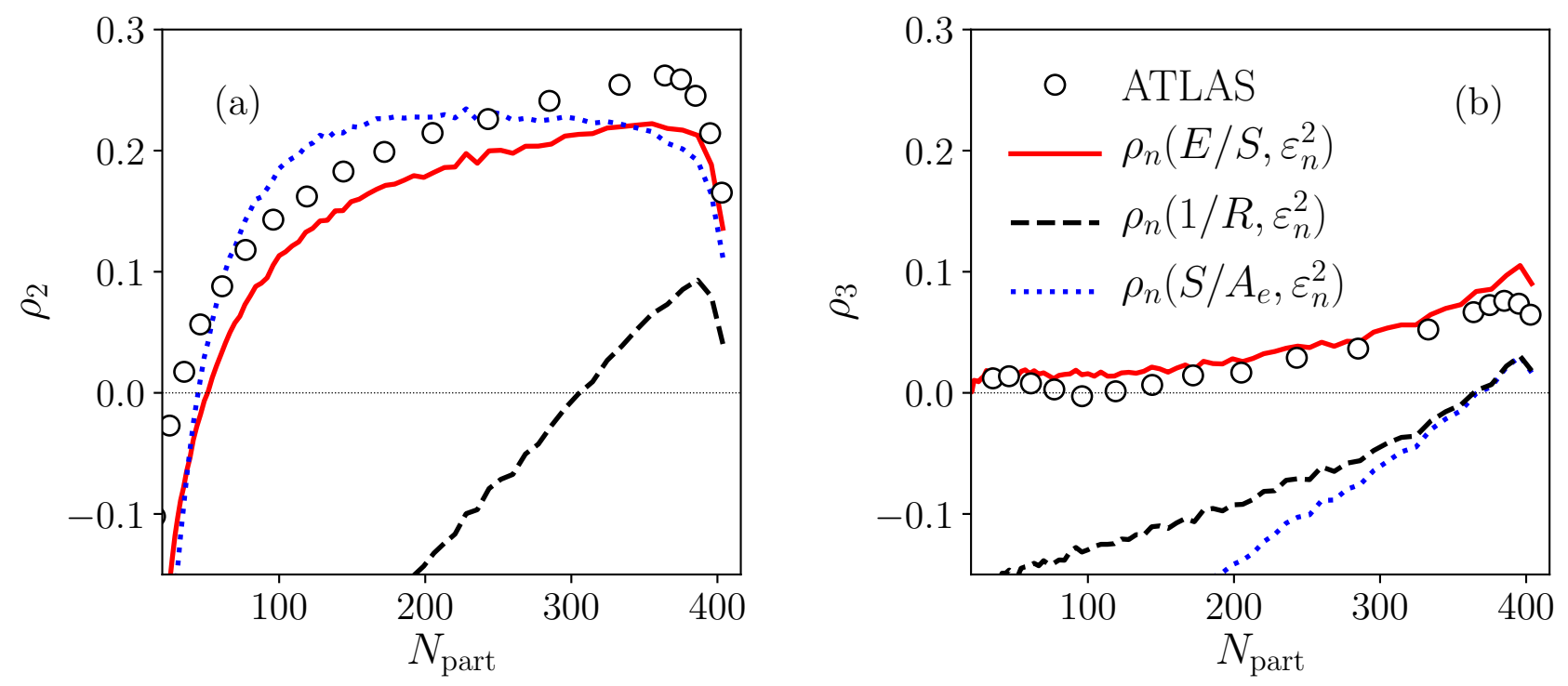

FIG. 3. (Color online) Variation of $\rho_{2}$ (a) and $\rho_{3}$ (b) with the number of participants in $\mathrm{Pb}+\mathrm{Pb}$ collisions at $\sqrt{s_{\mathrm{NN}}}=5.02 \mathrm{TeV}$. As in Fig. 1. symbols are experimental results from the ATLAS Collaboration [10. The shaded band is our result using Eq. (8). The width of the band is the statistical error evaluated through jackknife resampling. The dashed lines are obtained by replacing $E_{i} / S$ with $1 / R$ in Eq. $\left[8\right.$, and the dotted lines by replacing $E_{i} / S$ with $S / A_{e}$.

\section{CONCLUSIONS}

We have shown that ATLAS results on $\rho_{n}$ in $\mathrm{Pb}+\mathrm{Pb}$ collisions can be explained by hydrodynamics. The mechanism driving the correlation between the mean transverse momentum and anisotropic flow in $\mathrm{Pb}+\mathrm{Pb}$ collisions can be traced back to the initial density profile, i.e., to the early stages of the collision. This implies in turn that this observable has limited sensitivity to the details of the hydrodynamic expansion in general, and to the transport coefficients of the fluid in particular, as nicely confirmed by the hydrodynamic results (Fig. 9) of Ref. [36]. We have found that $\left\langle p_{t}\right\rangle$ fluctuations are driven by fluctuations of the initial energy over entropy ratio $E_{i} / S$, and not by the fluctuations of the fireball size as previously thought. By use of Eq. (8), models of initial conditions that fit anisotropic flow data and multiplicity fluctuations also naturally reproduce the centrality dependence of $\rho_{2}$ and $\rho_{3}$ measured by the ATLAS Collaboration without any further adjustment. Note that experimental data are also available for $\mathrm{p}+\mathrm{Pb}$ collisions, the study of which we leave for future work.

\section{ACKNOWLEDGMENTS}

FGG was supported by $\mathrm{CNPq}_{\text {(Conselho Nacional }}$ de Desenvolvimento Cientifico) grant 312932/2018-9, by INCT-FNA grant 464898/2014-5 and FAPESP grant 2018/24720-6. G.G. and J.-Y.O. were supported by USPCOFECUB (grant Uc Ph 160-16, 2015/13). J.N.H. ac- knowledges the support of the Alfred P. Sloan Foundation, support from the US-DOE Nuclear Science Grant No. de-sc0019175. J.-Y. O. thanks Piotr Bożek for discussions. We acknowledge useful discussions with Björn Schenke, Chun Shen, and Derek Teaney.

\section{Appendix A: Varying the parametrization of the initial profile}

We check the sensitivity of $\rho_{n}$, as defined by Eq. (8), to the parametrization of initial conditions. Figure 4 displays the variation of $\rho_{n}$ for three different values of $p$ in the $\mathrm{T}_{\mathrm{R}}$ ENTo model. Several qualitative trends are robust: $\rho_{2}$ and $\rho_{3}$ are both positive for central collisions; As the number of participants decreases from its maximum value, $\rho_{2}$ steeply increases and then decreases, eventually becoming negative, while the centrality dependence of $\rho_{3}$ is milder. But significant differences appear at the quantitative level, and the value $p=0$, which is the preferred value also for other observables [18, 28, agrees best with the recent $\rho_{n}$ data. We have also studied the dependence on the parameter $k$ governing the magnitude of fluctuations in $\mathrm{T}_{\mathrm{R}}$ ENTo. Results in Fig. 3 are obtained with $k=2$, but we have also carried out calculations with $k=1$, corresponding to larger fluctuations. We have found (not shown) that the results for $\rho_{2}$ are essentially unchanged except for a minor increase in central collisions, while the variation of $\rho_{3}$ becomes flatter, similar to the $p=-1$ results in Fig. 4 . 

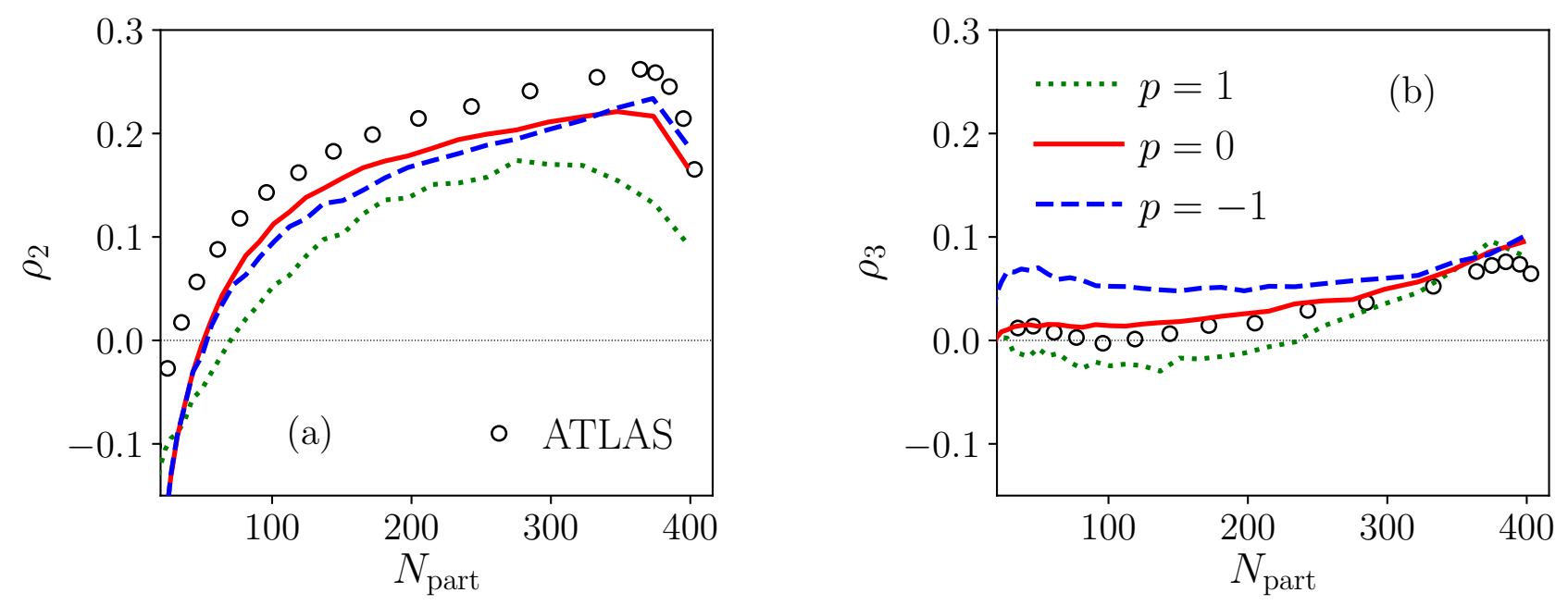

FIG. 4. (Color online) Dependence of $\rho_{2}$ (a) and $\rho_{3}$ (b) on the parameter $p$ in the $\mathrm{T}_{\mathrm{R}}$ ENTo model. Full lines: $p=0$ (entropy density $\left.s \propto \sqrt{T_{A} T_{B}}\right)$, as in Fig. 3 . Dotted lines: $p=1\left(s \propto T_{A}+T_{B}\right)$. Dashed lines: $p=-1\left(s \propto T_{A} T_{B} /\left(T_{A}+T_{B}\right)\right)$. We use broader centrality bins for this calculation than for Fig. 3 which explains the small differences between the $p=0$ results of the two figures. As in Figs. 1 and 3 symbols are ATLAS data [10.

[1] U. Heinz and R. Snellings, Ann. Rev. Nucl. Part. Sci. 63, 123-151 (2013) doi:10.1146/annurev-nucl-102212-170540 [arXiv:1301.2826 [nucl-th]].

[2] G. Aad et al. [ATLAS], JHEP 11, 183 (2013) doi:10.1007/JHEP11(2013)183 [arXiv:1305.2942 [hep$\mathrm{ex}]$.

[3] A. M. Sirunyan et al. [CMS], Phys. Lett. B 789, 643-665 (2019) doi:10.1016/j.physletb.2018.11.063 [arXiv:1711.05594 [nucl-ex]].

[4] S. Acharya et al. [ALICE], Phys. Rev. Lett. 123, no.14, $142301 \quad$ (2019) doi:10.1103/PhysRevLett.123.142301 [arXiv:1903.01790 [nucl-ex]].

[5] G. Aad et al. [ATLAS], Phys. Rev. C 90, no.2, $024905 \quad$ (2014) doi:10.1103/PhysRevC.90.024905 [arXiv:1403.0489 [hep-ex]].

[6] J. Adam et al. [ALICE], Phys. Rev. Lett. 117, 182301 (2016) doi:10.1103/PhysRevLett.117.182301 [arXiv:1604.07663 [nucl-ex]].

[7] A. M. Sirunyan et al. [CMS], Eur. Phys. J. C 80, no.6, 534 (2020) doi:10.1140/epjc/s10052-020-7834-9 [arXiv:1910.08789 [hep-ex]].

[8] S. Acharya et al. [ALICE], JHEP 05, 085 (2020) doi:10.1007/JHEP05(2020)085 [arXiv:2002.00633 [nucl$\mathrm{ex}]$.

[9] P. Bozek, Phys. Rev. C 93, no.4, 044908 (2016) doi:10.1103/PhysRevC.93.044908 [arXiv:1601.04513 [nucl-th]].

[10] G. Aad et al. [ATLAS], Eur. Phys. J. C 79, no.12, 985 (2019) doi:10.1140/epjc/s10052-019-7489-6 [arXiv:1907.05176 [nucl-ex]].

[11] P. Bozek and H. Mehrabpour, Phys. Rev. C 101, no.6, 064902 (2020) doi:10.1103/PhysRevC.101.064902 [arXiv:2002.08832 [nucl-th]].

[12] G. Giacalone, J. Noronha-Hostler, M. Luzum and J. Y. Ollitrault, Phys. Rev. C 97, no.3, 034904 (2018) doi:10.1103/PhysRevC.97.034904 [arXiv:1711.08499 [nucl-th]].

[13] B. Alver et al. [PHOBOS], Phys. Rev. Lett. 98, 242302 (2007) doi:10.1103/PhysRevLett.98.242302 [arXiv:nuclex/0610037 [nucl-ex]].

[14] B. Alver and G. Roland, Phys. Rev. C 81, 054905 (2010) [erratum: Phys. Rev. C 82, 039903 (2010)] doi:10.1103/PhysRevC.82.039903 [arXiv:1003.0194 [nucl-th]].

[15] D. Teaney and L. Yan, Phys. Rev. C 83, 064904 (2011) doi:10.1103/PhysRevC.83.064904 [arXiv:1010.1876 [nucl-th]].

[16] F. G. Gardim, F. Grassi, M. Luzum and J. Y. Ollitrault, Phys. Rev. C 85, 024908 (2012) doi:10.1103/PhysRevC.85.024908 [arXiv:1111.6538 [nucl-th]].

[17] H. Niemi, G. S. Denicol, H. Holopainen and P. Huovinen, Phys. Rev. C 87, no.5, 054901 (2013) doi:10.1103/PhysRevC.87.054901 [arXiv:1212.1008 [nucl-th]].

[18] G. Giacalone, J. Noronha-Hostler and J. Y. Ollitrault, Phys. Rev. C 95, no.5, 054910 (2017) doi:10.1103/PhysRevC.95.054910 [arXiv:1702.01730 [nucl-th]].

[19] W. Broniowski, M. Chojnacki and L. Obara, Phys. Rev. C 80, 051902 (2009) doi:10.1103/PhysRevC.80.051902 [arXiv:0907.3216 [nucl-th]].

[20] P. Bozek and W. Broniowski, Phys. Rev. C 85, $044910 \quad$ (2012) doi:10.1103/PhysRevC.85.044910 [arXiv:1203.1810 [nucl-th]].

[21] P. Bożek and W. Broniowski, Phys. Rev. C 96, no.1, 014904 (2017) doi:10.1103/PhysRevC.96.014904 [arXiv:1701.09105 [nucl-th]].

[22] F. G. Gardim, G. Giacalone, M. Luzum and J. Y. Ollitrault, Nucl. Phys. A 1005, 121999 (2021) doi:10.1016/j.nuclphysa.2020.121999 [arXiv:2002.07008 [nucl-th]]. 
[23] J. Adams et al. [STAR], Phys. Rev. C 72, 044902 (2005) doi:10.1103/PhysRevC.72.044902 [arXiv:nuclex/0504031 [nucl-ex]].

[24] J. Noronha-Hostler, G. S. Denicol, J. Noronha, R. P. G. Andrade and F. Grassi, Phys. Rev. C 88, no.4, 044916 (2013) doi:10.1103/PhysRevC.88.044916 [arXiv:1305.1981 [nucl-th]].

[25] J. Noronha-Hostler, J. Noronha and F. Grassi, Phys. Rev. C 90, no.3, $034907 \quad$ (2014) doi:10.1103/PhysRevC.90.034907 [arXiv:1406.3333 [nucl-th]].

[26] J. Noronha-Hostler, J. Noronha and M. Gyulassy, Phys. Rev. C 93, no.2, 024909 (2016) doi:10.1103/PhysRevC.93.024909 [arXiv:1508.02455 [nucl-th]].

[27] J. S. Moreland, J. E. Bernhard and S. A. Bass, Phys. Rev. C 92, no.1, $011901 \quad$ (2015) doi:10.1103/PhysRevC.92.011901 [arXiv:1412.4708 [nucl-th]].

[28] J. E. Bernhard, J. S. Moreland, S. A. Bass, J. Liu and U. Heinz, Phys. Rev. C 94, no.2, 024907 (2016) doi:10.1103/PhysRevC.94.024907 [arXiv:1605.03954 [nucl-th]].

[29] J. Vredevoogd and S. Pratt, Phys. Rev. C 79, 044915 (2009) doi:10.1103/PhysRevC.79.044915 [arXiv:0810.4325 [nucl-th]].

[30] W. van der Schee, P. Romatschke and S. Pratt, Phys. Rev. Lett. 111, no.22, $222302 \quad$ (2013) doi:10.1103/PhysRevLett.111.222302 [arXiv:1307.2539 [nucl-th]].

[31] A. Kurkela, A. Mazeliauskas, J. F. Paquet, S. Schlichting and D. Teaney, Phys. Rev. Lett. 122, no.12, 122302 (2019) doi:10.1103/PhysRevLett.122.122302 [arXiv:1805.01604 [hep-ph]].

[32] P. F. Kolb, P. Huovinen, U. W. Heinz and H. Heiselberg, Phys. Lett. B 500, 232-240 (2001) doi:10.1016/S03702693(01)00079-X [arXiv:hep-ph/0012137 [hep-ph]].

[33] S. Borsanyi, Z. Fodor, C. Hoelbling, S. D. Katz, S. Krieg and K. K. Szabo, Phys. Lett. B 730, 99-104 (2014) doi:10.1016/j.physletb.2014.01.007 [arXiv:1309.5258 [hep-lat]].

[34] P. Alba, V. Mantovani Sarti, J. Noronha, J. NoronhaHostler, P. Parotto, I. Portillo Vazquez and C. Ratti, Phys. Rev. C 98, no.3, 034909 (2018) doi:10.1103/PhysRevC.98.034909 [arXiv:1711.05207 [nucl-th]].

[35] D. Teaney, Phys. Rev. C 68, 034913 (2003) doi:10.1103/PhysRevC.68.034913 [arXiv:nuclth/0301099 [nucl-th]].

[36] B. Schenke, C. Shen and D. Teaney, Phys. Rev. C 102, no.3, 034905 (2020) doi:10.1103/PhysRevC.102.034905 [arXiv:2004.00690 [nucl-th]].

[37] A. Bazavov et al. [HotQCD], Phys. Lett. B 795, 15-21 (2019) doi:10.1016/j.physletb.2019.05.013 [arXiv:1812.08235 [hep-lat]].

[38] B. Schenke, S. Jeon and C. Gale, Phys. Rev. C 82, 014903 (2010) doi:10.1103/PhysRevC.82.014903 [arXiv:1004.1408 [hep-ph]].

[39] B. Schenke, S. Jeon and C. Gale, Phys. Rev. Lett. 106, 042301 (2011) doi:10.1103/PhysRevLett.106.042301 [arXiv:1009.3244 [hep-ph]].

[40] J. F. Paquet, C. Shen, G. S. Denicol, M. Luzum, B. Schenke, S. Jeon and C. Gale, Phys. Rev. C 93, no.4, 044906 (2016) doi:10.1103/PhysRevC.93.044906 [arXiv:1509.06738 [hep-ph]].

[41] F. G. Gardim, G. Giacalone, M. Luzum and J. Y. Ollitrault, Nature Phys. 16, no.6, 615-619 (2020) doi:10.1038/s41567-020-0846-4 [arXiv:1908.09728 [nuclth]].

[42] F. G. Gardim, F. Grassi, M. Luzum and J. NoronhaHostler, Phys. Rev. C 95, no.3, 034901 (2017) doi:10.1103/PhysRevC.95.034901 [arXiv:1608.02982 [nucl-th]].

[43] H. Heiselberg and A. M. Levy, Phys. Rev. C 59, 27162727 (1999) doi:10.1103/PhysRevC.59.2716 [arXiv:nuclth/9812034 [nucl-th]].

[44] L. He, T. Edmonds, Z. W. Lin, F. Liu, D. Molnar and F. Wang, Phys. Lett. B 753, 506-510 (2016) doi:10.1016/j.physletb.2015.12.051 [arXiv:1502.05572 [nucl-th]]. 